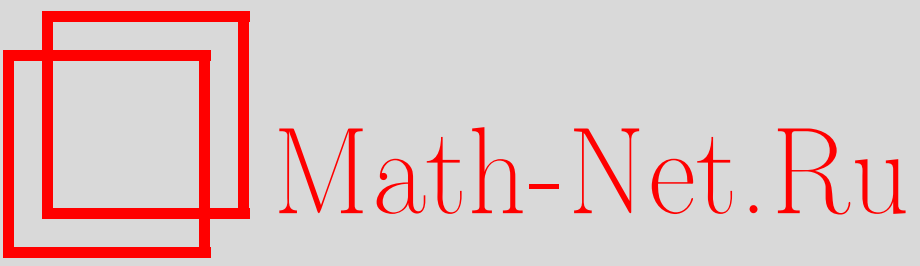

Е. Грбич, С. Терио, Гомотопический тип дополнения конфигурации координатных подпространств коразмерности два, УМН, 2004, том 59, выпуск 6, 203-204

DOI: https://doi.org/10.4213/rm803

Использование Общероссийского математического портала Math-Net.Ru подразумевает, что вы прочитали и согласны с пользовательским соглашением

http://www.mathnet.ru/rus/agreement

Параметры загрузки:

IP: 34.227 .88 .159

26 апреля 2023 г., 12:21:25 


\title{
ГОМОТОПИЧЕСКИЙ ТИП ДОПОЛНЕНИЯ КОНФИГУРАЦИИ КООРДИНАТНЫХ ПОДПРОСТРАНСТВ КОРАЗМЕРНОСТИ ДВА
}

\author{
Е. ГрБич, С. ТЕРио
}

Координатное подпространство в $\mathbb{C}^{n}$ есть $L_{\sigma}=\left\{\left(z_{1}, \ldots, z_{n}\right) \in \mathbb{C}^{n} \mid z_{i_{1}}=\cdots=z_{i_{k}}=0\right\}$, где $\sigma=\left\{i_{1}, \ldots, i_{k}\right\}$ - подмножество в [n]. Каждому симплициальному комплексу $K$ на множестве $[n]$ сопоставим конфигурацию координатных подпространств $\mathscr{C} \mathscr{A}(K)=\left\{L_{\sigma} \mid \sigma \notin K\right\}$ и ее дополнение $U(K)=\mathbb{C}^{n} \backslash \bigcup_{\sigma \notin K} L_{\sigma}$. С другой стороны, комплексу $K$ сопоставляется пространство Дэвиса-Янушкиевича $D J(K)=\bigcup_{\sigma \in K} B T_{\sigma} \subset B T^{n}$, где $B T^{n}$ - классифицирующее пространство $n$-мерного тора, т.е. произведение $n$ экземпляров пространства $\mathbb{C} P^{\infty}$, и $B T_{\sigma}:=$ $\left\{\left(x_{1}, \ldots, x_{n}\right) \in B T^{n} \mid x_{i}=*\right.$, где $\left.i \notin \sigma\right\}$. Определим $\mathscr{Z}_{K}$ как гомотопический слой вложения $D J(K) \rightarrow B T^{n}$. Согласно резултату $[1 ; 8.9]$, имеется эквивариантная деформационная ретракция $U(K) \rightarrow \mathscr{Z}_{K}$, кроме того, кольцо целочисленных когомологий пространства $\mathscr{Z}_{K}$ было вычислено в $[1 ; 7.6,7.7]$.

Теорема 1. Дополнение конфигурации координатных подпространств коразмерности два в $\mathbb{C}^{n}$ гомотопически әквивалентно букету сфер $\bigvee_{k=2}^{n}(k-1)\left(\begin{array}{l}n \\ k\end{array}\right) S^{k+1}$.

ДоКАЗАТЕЛЬСтво. Пусть $K$ - несвязное объединение $n$ точек. Тогда $D J(K)$ является букетом из $n$ копий пространства $\mathbb{C} P^{\infty}$, а $U(K)$ получается удалением из $\mathbb{C}^{n}$ всех координатных подпространств коразмерности два, т.е. вида $z_{i}=z_{j}=0,1 \leqslant i<j \leqslant n$. Таким образом, для доказательства теоремы необходимо описать гомотопический слой вложения $\bigvee_{t=1}^{n} \mathbb{C} P^{\infty} \rightarrow$ $\prod_{t=1}^{n} \mathbb{C} P^{\infty}$. Необходимое описание получается применением предложения 1 к случаю $X_{1}=$ $\cdots=X_{n}=\mathbb{C} P^{\infty}$, с учетом того, что $\Omega \mathbb{C} P^{\infty} \simeq S^{1}$.

Необходимо подчеркнуть, что теорема 1 имеет место без применения надстройки. Ранее известные гомотопические разложения имели место лишь после применения некоторого числа надстроек. Наилучшее из таких разложений принадлежит Шаперу [2] и требует одной надстройки. Для завершения доказательства теоремы 1 необходимо доказать предложение 1 . Это утверждение было впервые доказано Портером [3] путем исследования подпространств в стягиваемых пространствах. Мы приводим более эффективное доказательство, основанное на лемме о кубе. Мы работаем в категории связных пространств с отмеченной точкой и непрерьвных отображений. Пусть $*$ обозначает отмеченную точку. Для пары пространств $X, Y$ положим $X \rtimes Y=$ $(X \times Y) /(* \times Y), X \wedge Y=(X \rtimes Y) /(X \times *)$ и $X * Y=\Sigma X \wedge Y$. Будем обозначать тождественное отображение на $X$ через $X$ и отображение в точку через $*$.

Лемма 2. Пусть $A, B$ и $C$ - пространства, а $Q$ - гомотопический копредел отображения $A \times B \stackrel{* \times B}{\longrightarrow} C \times B$ и проекиии $A \times B \stackrel{\pi_{1}}{\longrightarrow} A$. Тогда $Q \simeq(A * B) \vee(C \rtimes B)$.

ДокАЗАТЕЛЬСтво. Рассмотрим диаграмму итерированных гомотопических копределов

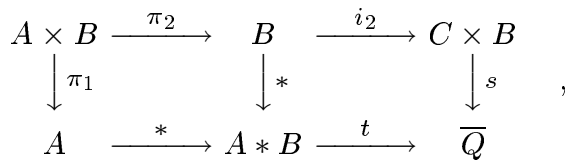

где $\pi_{2}$ - проекция, а $i_{2}$ - вложение. Известно, что левьй квадрат является гомотопически кодекартовым. Пространство $\bar{Q}$ определяется как гомотопический копредел правого квадрата. Заметим, что $i_{2} \circ \pi_{2} \simeq * \times B$. Внешний прямоугольник является гомотопически кодекартовым, как композиция двух гомотопически кодекартовых квадратов, поэтому $\bar{Q} \simeq Q$. Из правого квадрата вытекает, что гомотопическим кослоем отображения $C \times B \rightarrow Q$ является $\Sigma B \vee(A * B)$. Следовательно, отображение $t$ имеет левое гомотопически обратное $r$. Кроме того, $s \circ i_{2} \simeq *$, и поэтому стягивание $B$ в точку в правом квадрате приводит к гомотопическому корасслоению $C \rtimes B \longrightarrow Q \stackrel{r}{\longrightarrow} A * B$, где $r \circ t$ гомотопно тождественному отображению.

Лемма 3. Для любьх $Y_{1}, \ldots, Y_{n}$ имеет место гомотопическая әквивалентность

$$
\Sigma\left(Y_{1} \times \cdots \times Y_{n}\right) \simeq \bigvee_{k=1}^{n}\left(\bigvee_{1 \leqslant i_{1}<\cdots<i_{k} \leqslant n} \Sigma Y_{i_{1}} \wedge \cdots \wedge Y_{i_{k}}\right)
$$


ДокАЗАТЕльство. Индуктивное применение разложения $\Sigma(A \times B)=\Sigma A \vee \Sigma B \vee(\Sigma A \wedge B)$. Следующее утверждение принадлежит Матеру [4] и известно как лемма о кубе.

ЛЕмма 4. Пусть задана диаграмма пространств и отображений

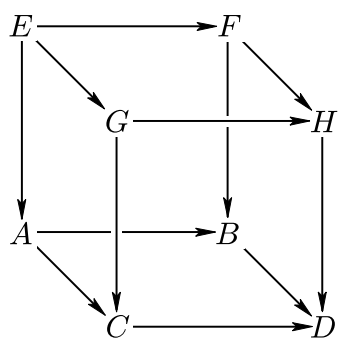

где нижняя грань является гомотопически кодекартовым квадратом, а боковые стороны являются декартовыми квадратами, индуцированными отображением $H \rightarrow D$. Тогда верхняя грань является гомотопически кодекартовым квадратом.

ПРЕДЛОЖЕНИЕ 5. Пусть $X_{1}, \ldots, X_{n}$ - пространства и $F_{n}$ - гомотопический слой вложения $X_{1} \vee \cdots \vee X_{n} \rightarrow X_{1} \times \cdots \times X_{n}$. Тогда имеется гомотопическое разложение

$$
F_{n} \simeq \bigvee_{k=2}^{n}\left(\bigvee_{1 \leqslant i_{1}<\cdots<i_{k} \leqslant n}(k-1)\left(\Sigma \Omega X_{i_{1}} \wedge \cdots \wedge \Omega X_{i_{k}}\right)\right)
$$

ДокАЗАТЕЛЬСТВо. Применим индукцию по $n$. При $n=2$ хорошо известно, что $F_{2} \simeq$ $\Sigma \Omega X_{1} \wedge \Omega X_{2}$. Пусть $n \geqslant 3$ и предположим, что утверждение имеет место для $F_{n-1}$. Положим $M_{k}=X_{1} \vee \cdots \vee X_{k}$ и $N_{k}=X_{1} \times \cdots \times X_{k}$. Заметим, что $M_{n}$ является копроизведением $M_{n-1}$ и $X_{n}$. Отображая каждую вершину соответствуюшего кодекартова квадрата в $N_{n}$, мы получаем гомотопические расслоения $\Omega N_{n} \rightarrow * \rightarrow N_{n}, \Omega N_{n-1} \rightarrow X_{n} \rightarrow N_{n}, F_{n-1} \times$ $\Omega X_{n} \rightarrow M_{n-1} \rightarrow N_{n}$ и $F_{n} \rightarrow M_{n} \rightarrow N_{n}$. Представим $N_{n}$ как $N_{n-1} \times X_{n}$. Тогда из леммы 4 вытекает существование гомотопически кодекартова квадрата

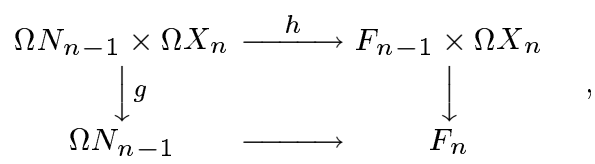

где, как легко видеть, $g$ является проекцией, а $h$ - связывающим отображением для гомотопического расслоения $F_{n-1} \times \Omega X_{n} \rightarrow M_{n-1} \times * \rightarrow N_{n-1} \times X_{n}$. Следовательно, $h \simeq \partial_{n-1} \times \Omega X_{n}$, где $\partial_{n-1}-$ связьвающее отображение для расслоения $F_{n-1} \rightarrow M_{n-1} \rightarrow N_{n-1}$. Но $\partial_{n-1} \simeq *$, так как отображение $\Omega M_{n-1} \rightarrow \Omega N_{n-1}$ имеет правое гомотопически обратное. Итак, $h \simeq * \times$ $\Omega X_{n}$. В силу леммы $2, F_{n} \simeq\left(\Omega N_{n-1} * \Omega X_{n}\right) \vee\left(F_{n-1} \rtimes \Omega X_{n}\right)$. Поскольку пространство $F_{n-1}$ является надстройкой, мы имеем $F_{n-1} \rtimes \Omega X_{n} \simeq F_{n-1} \vee\left(F_{n-1} \wedge \Omega X_{n}\right)$. Теперь, применяя разложение $\Sigma \Omega N_{n} \simeq \Sigma\left(\Omega X_{1} \times \cdots \times \Omega X_{n}\right)$ из леммы 3 совместно с разложением для $F_{n-1}$ из предположения индукции и приводя подобные члены, мы получаем необходимое разложение пространства $F_{n}$ в букет.

Авторы выражают благодарность Т.Е. Панову и Н. Рэю за стимулирующие обсуждения, привлекшие нас к рассмотрению этой проблемы.

\section{СПИСОК ЛИТЕРАТУРЫ}

[1] V. M. Buchstaber, T. E. Panov // Univ. Lecture Ser. 2002. V. 24. [2] Ch. Schaper // Math. Ann. 1997. V. 309. № 3. P. 463-473. [3] T. Porter // Amer. J. Math. 1966. V. 88. P. 655663. [4] M. Mather // Canad. J. Math. 1976. V. 28. № 2. P. 225-263.

University of Aberdeen, United Kingdom

Принято редколлегией E-mail: jelena@maths.abdn.ac.uk, 27.07 .2004

s.theriault@maths.abdn.ac.uk 